Cite this: Nanoscale, 2013, 5, 953

\title{
2D to 3D crossover of the magnetic properties in ordered arrays of iron oxide nanocrystals $t$
}

\author{
Bertrand Faure, $\neq^{a}$ Erik Wetterskog, $\ddagger^{a}$ Klas Gunnarsson, ${ }^{b}$ Elisabeth Josten, ${ }^{c}$ \\ Raphaël P. Hermann, ${ }^{\text {cd }}$ Thomas Brückel, ${ }^{c}$ Jens Wenzel Andreasen, ${ }^{e}$ Florian Meneau, ${ }^{f}$ \\ Mathias Meyer, ${ }^{9}$ Alexander Lyubartsev, ${ }^{a}$ Lennart Bergström, ${ }^{a}$ German Salazar- \\ Alvarez $^{a}$ and Peter Svedlindh ${ }^{* b}$
}

\begin{abstract}
The magnetic 2D to 3D crossover behavior of well-ordered arrays of monodomain $\gamma-\mathrm{Fe}_{2} \mathrm{O}_{3}$ spherical nanoparticles with different thicknesses has been investigated by magnetometry and Monte Carlo (MC) simulations. Using the structural information of the arrays obtained from grazing incidence small-angle X-ray scattering and scanning electron microscopy together with the experimentally determined values for the saturation magnetization and magnetic anisotropy of the nanoparticles, we show that $M C$ simulations can reproduce the thickness-dependent magnetic behavior. The magnetic dipolar particle interactions induce a ferromagnetic coupling that increases in strength with decreasing thickness of the array. The $2 \mathrm{D}$ to $3 \mathrm{D}$ transition in the magnetic properties is mainly driven by a change in the orientation of the magnetic vortex states with increasing thickness, becoming more isotropic as the thickness of the array increases. Magnetic anisotropy prevents long-range ferromagnetic order from being established at low temperature and the nanoparticle magnetic moments instead freeze along directions defined by the distribution of easy magnetization directions.
\end{abstract}

Received 2nd October 2012 Accepted 27th November 2012

DOI: 10.1039/c2nr33013j

www.rsc.org/nanoscale short separation distance between the nanocrystals and the geometric arrangement of the arrays promote dipolar interactions and affect electron transport properties. ${ }^{6-9}$

The characterization of arrays of magnetic nanoparticles assembled into different structures has been reported in several studies. ${ }^{10-18}$ One-dimensional (1D) structures consisting of comparatively large nanoparticles with blocked magnetic moments can be formed with the aid of external magnetic fields or by using molecular linkers. It was shown that dipolar interactions in the chain-like structures result in a higher blocking temperature than that of isolated nanoparticles. ${ }^{\mathbf{1 9 2} 0}$

Moving to two-dimensional (2D) and three-dimensional (3D) arrays, the seminal theoretical work of Luttinger and Tisza established that the magnetic order of dipolar arrays on cubic lattices is strongly dependent on the lattice symmetry. ${ }^{21}$ More recent theoretical work has investigated the magnetic properties of 2D arrays of magnetic nanoparticles with different array structures, sizes and shapes. ${ }^{22,23}$ In the absence of magnetic anisotropy, vortex and flux closure states are predicted in the thermodynamic limit of an infinite number of magnetic nanoparticles in a $2 \mathrm{D}$ array, ${ }^{23}$ and confirmed experimentally using magnetic force microscopy and spectroscopy and electron holography. ${ }^{\mathbf{1 4 , 1 7}}$ In arrays of magnetic nanoparticles with strong magnetocrystalline anisotropy, experiments and simulations reveal a competition between the magnetic anisotropy and the dipolar interactions, inducing an increase in short-range ferromagnetic ordering with the temperature. ${ }^{16}$ Macroscopic 3D 
$f c c$ crystals of magnetoferritin, a protein-nanoparticle hybrid, exhibit a blocking temperature much higher than that of a randomly packed configuration of nanoparticles. ${ }^{18}$

Although the studies on 2D and 3D systems described above have given an important insight into the collective magnetic properties of nanoparticle arrays, the crossover from the $2 \mathrm{D}$ to $3 \mathrm{D}$ behavior is still poorly understood and experimental or theoretical studies on structurally well-ordered arrays are sparse. For instance, quasi-ordered layered structures prepared by sequential deposition of Co and $\mathrm{Co}-\mathrm{Al}_{2} \mathrm{O}_{3}$ have been studied by $\mathrm{AC}$ susceptibility measurements. ${ }^{24}$ It was found that the effective energy barrier increases with increasing number of $\mathrm{Co}-$ $\mathrm{Al}_{2} \mathrm{O}_{3}$ bilayers and that the $3 \mathrm{D}$ limit for collective dynamics was realized at five bilayers.

The magnetic properties of arrays of superparamagnetic nanoparticles can also be studied by Monte Carlo (MC) simulations, with a straightforward account of the temperature. ${ }^{25,26}$ In stacked layers of small particles the effects of the number of layers, the coordination number and the distance between the nanoparticles on the remanent magnetization have been studied with MC simulations. ${ }^{27}$ It was found that the tendency of nanoparticle magnetic moments to form closed-circuit alignments increased with the number of layers, suggesting a transition from the $2 \mathrm{D}$ to $3 \mathrm{D}$ behavior. Although some information on the $2 \mathrm{D}$ to $3 \mathrm{D}$ crossover has been obtained from $\mathrm{MC}$ simulations, no experimental study of the 2D to 3D transition of the magnetic behavior has been presented for large wellordered arrays.

In this work, we have studied the structural and magnetic properties of ordered $\gamma-\mathrm{Fe}_{2} \mathrm{O}_{3}$ nanoparticle arrays with varying thickness. The structure of the arrays has been determined by a combination of grazing-incidence small-angle X-ray scattering (GISAXS) and scanning electron microscopy (SEM). The magnetic behavior of the arrays was investigated with temperature- and field-dependent zero-field-cooled (ZFC) and fieldcooled (FC) magnetization measurements, as well as with temperature- and frequency-dependent AC susceptibility measurements. Furthermore, MC simulations have been performed to explore the temperature-dependence of the ZFC magnetization and the influence of the thickness of the arrays on the remanent magnetization. Experiments and simulation results reveal thickness-dependent dipolar couplings in the arrays. The mechanism of the $2 \mathrm{D}$ to $3 \mathrm{D}$ transition is interpreted as a change in the vorticity of the magnetization vector field.

\section{Materials and methods}

Monodisperse iron oxide nanoparticles were synthesized by a modified thermal decomposition route, ${ }^{28,29}$ and separated from the mother liquor (see ESI $\dagger$ for details). According to transmission electron microscopy (TEM) measurements, the obtained iron oxide nanoparticles are spheroidal with a particle diameter of $9.1 \pm 0.6 \mathrm{~nm}$ and a composition near that of maghemite $\left(\gamma-\mathrm{Fe}_{2} \mathrm{O}_{3}\right)$, in good agreement with a previous report. $^{30}$

Arrays of iron oxide nanospheres were deposited on silicon wafers $\left(5 \times 5 \mathrm{~mm}^{2}\right)$ by drop casting of dispersions of different concentrations in an external magnetic field applied perpendicular to the substrate. ${ }^{29}$ The concentrations were adjusted to give average nominal thicknesses of 25 and $60 \mathrm{~nm}$ for the two most dilute dispersions, referred to as the thin and medium arrays, respectively. The drying of the most concentrated dispersion in a magnetic field resulted in the substrate being partially covered by bulk-like islands of a few $\mu \mathrm{m}$ in thickness, referred to as the thick array. For comparison, a dilute dispersion of nanospheres (with a nanoparticle concentration of $0.1 \mathrm{wt} \%$ ), referred to as the dispersion was also characterized by small angle X-ray scattering (SAXS) and dynamic light scattering (DLS) (see ESI $\dagger$ for details).

The GISAXS measurements on the thin array were carried out on the SWING beamline at the SOLEIL synchrotron in Saclay, France. A CCD detector was located in an evacuated tank at a distance of $0.9 \mathrm{~m}$ from the sample. The incident wavelength was set to $1.77 \AA$ and the critical angle between the primary beam and the sample surface was $0.4^{\circ}$. The other GISAXS measurements were performed on a laboratory setup at the Technical University of Denmark, Risø, using a $\mathrm{Cu}-\mathrm{K} \alpha$ source. The detector distance was $1.44 \mathrm{~m}$ with an incident angle of $0.4^{\circ}{ }^{31}$

$\mathrm{AC}$ and DC magnetization measurements were performed in a Quantum Design MPMS-XL SQUID magnetometer. Magnetization versus temperature was studied between $5 \mathrm{~K}$ and $300 \mathrm{~K}$, following two different protocols. The ZFC magnetization was obtained by cooling the sample to $5 \mathrm{~K}$ in zero field, turning on a weak magnetic field and measuring the magnetization as the sample warmed up. The FC magnetization was subsequently obtained by measuring the magnetization, in the same applied field, as the sample cooled down to $5 \mathrm{~K}$. The temperature range used for the frozen dispersion was 5-250 K. The ZFC/FC measurements were performed with different applied magnetic fields in the range $0.4-8 \mathrm{kA} \mathrm{m}^{-1}$. Isothermal magnetization measurements were performed at $10 \mathrm{~K}$; the magnetization versus applied field was measured in the field range $\pm 800 \mathrm{kA}$ $\mathrm{m}^{-1}$. The sample weights correlate well with the measured saturation magnetic moments of the arrays and were used to normalize the magnetic measurements. In all measurements the external field was applied in-plane of the substrate.

MC simulations were performed on a distorted $f c c$ lattice of magnetic dipole moments using the lattice parameters of the thick array (Table 1). The thin and medium samples were simulated with 3 and $6 f c c$-stacked hexagonal layers of dipoles, respectively. A thick finite array (30 layers) and a bulk array were simulated to study the thick sample. A hexagonal monolayer of dipoles has been studied as well, in order to investigate the magnetic behavior of an ideal $2 \mathrm{D}$ array. The properties of the

Table 1 Structural characteristics of the samples as determined from the GISAXS measurements. The unit cells correspond to a $R \overline{3} m$ lattice, space group no. 146.

\begin{tabular}{lllc}
\hline Sample & $a(\mathrm{~nm})$ & $c(\mathrm{~nm})$ & $\begin{array}{l}\text { In-plane correlation } \\
\text { length }(\mathrm{nm})\end{array}$ \\
\hline Thin array & $12.8 \pm 1.0$ & $27.3 \pm 1.0$ & $42 \pm 10$ \\
Medium array & $12.6 \pm 0.6$ & $27.3 \pm 1.4$ & $67 \pm 30$ \\
Thick array & $12.1 \pm 0.4$ & $30.0 \pm 2.0$ & $100 \pm 20$
\end{tabular}


dispersion were simulated on an array of non-interacting dipoles. The temperature-dependent magnetization of the particles was calculated using the parameters of the thick arrays reported in Table 2, and the anisotropy constant measured on the dispersion (see ESI $\dagger$ ). The magnetic easy axes of the particles were chosen randomly at the beginning of the simulation and kept constant (see ESI $\dagger$ ). The dipole moments are initially oriented along the easy axes (for the simulated ZFC data) or along the external field (for the simulated remanent magnetization) before reorientations of the moments are performed using the Metropolis algorithm. The energy calculation includes the contributions of the external field, the uniaxial magnetic anisotropy and the dipolar interactions. ${ }^{27,32}$ The magnetization of the simulated arrays was thermally averaged over 500 configurations after reaching thermodynamic equilibrium. The thin, medium and thick arrays were simulated with $2 \mathrm{D}$ periodic boundary conditions, using the minimum image convention, while the bulk arrays were simulated with $3 \mathrm{D}$ periodic boundary conditions. The ZFC magnetization of the dispersion was simulated by turning off the dipolar interactions.

The presence and orientation of magnetic vortex states were quantified from the vorticity of the magnetic moments $(\vec{V}=\nabla \times$ $\vec{m})$. The root sum square of the vorticity vectors increases with the extent of non-collinear and anti-parallel magnetic states. In addition, the vorticity vector can be split into an in-plane component relating to magnetic loops in the planes parallel to the substrate (vortex states in the $x, y$-plane generate vorticity vectors along the $z$-axis, with an average magnitude $\left\langle V_{\text {in }}\right\rangle=\frac{1}{N_{\text {box }}} \sum \sqrt{V_{\mathrm{z}}^{2}}, N_{\text {box }}$ being the number of dipoles in the simulation box) and an out-of-plane component (the average root sum square of the vorticity along the $x$ and $y$ axes, $\left.\left\langle V_{\text {out }}\right\rangle=\frac{1}{N_{\text {box }}} \sum\left(\sqrt{V_{x}^{2}+V_{y}^{2}}\right)\right)$. The average orientation of the dipoles was estimated from the ratio of twice the squared inplane contribution over the squared out-of-plane one. This ratio has a value of 1 for an isotropic distribution of vorticity vectors.

\section{Results and discussion}

Previous reports ${ }^{24}$ have indicated that large changes in magnetic properties due to thickness effects should primarily be observed for relatively thin magnetic films. Therefore in order to investigate the $2 \mathrm{D}$ to $3 \mathrm{D}$ crossover effects in the nanoparticle assemblies, the magnetic properties of the two thin arrays were compared to that of the thick bulk-like array and the dilute dispersion (see Materials and methods). A hexagonal symmetry of the top layer is evident from the Fast Fourier Transforms (FFT) of the SEM images in all cases (see Fig. 1). The GISAXS pattern of the thin array (Fig. 1(d)) displays broad peaks along the $Q_{z}$ direction, confirming that it is a thin layer. The relatively sharp peaks in $Q_{y}$ indicate long range inplane correlations. ${ }^{33}$ The unit cell parameters and the corresponding nearest neighbor distances could be computed by assuming a $16 \mathrm{~mm}$ plane group of the first layer. GISAXS patterns of the medium and thick arrays (Fig. 1(e) and (f)) display Bragg spots, indicating a long range order ${ }^{34}$ A trigonal unit cell $(R \overline{3} m$, space group 146) was determined from the analysis, corresponding to a $f c c$ lattice with a $1 \%$ distortion in the $\langle 111\rangle$ direction. The in-arc-direction smeared Bragg spots suggest a small spread in the array tilt, probably arising during the drying of the film. ${ }^{34}$ The geometric relationship between the primitive rhombohedral and $f c c$ unit cells is illustrated in Fig. 1(g) and a summary of the unit cell parameters and the derived order parameters are given in Table 1 . The lattice parameters and interparticle distances agree well with the observed nanoparticle size (see ESI $\dagger$ ) and the thickness of the oleic acid capping layer. Moreover, we notice that the in-plane correlation length and hence the order of the arrays increases with increasing array thickness. An increasing surface roughness is also observed with increasing array thickness, as seen in atomic force microscopy (AFM) images (see ESI + ) and is likely due to dewetting effects during the final drying stages of the dropcasting. ${ }^{35}$ Furthermore, the thick array consists of domains with thickness of a few $\mu \mathrm{m}$, making it a good representation of a bulk-like system (see Fig. 1(c) and ESI $\dagger$ ).

The field dependence of the magnetization of the thin and thick arrays, and of the dilute dispersion $(0.1 \mathrm{wt} \%$ in cyclohexane), is shown in Fig. 2 where the low-field region has been enlarged to highlight the variations in the remanent magnetization. We find that the remanent magnetization of the dispersion is smaller than that of the arrays suggesting that the dipolar couplings, which are stronger in the arrays than in the dispersion, induce a net ferromagnetic interaction. Interestingly, the strength of the ferromagnetic couplings in the arrays decreases with increasing thickness, an effect that was investigated further by temperature dependent ZFC and FC magnetization measurements.

The in-plane ZFC/FC susceptibility data (shown in Fig. 3(a)) further corroborate the dipolar induced ferromagnetic-like

Table 2 Fitted values of the zero-temperature saturation magnetization $M_{s}(0)$ (experimental) and the mean-field constant $\alpha$ (experimental and simulated). The experimental values are extracted by fitting the expression in eqn (1) to the measurements performed in an external field of $0.4 \mathrm{kA} \mathrm{m}^{-1}$ between $150 \mathrm{~K}$ and $300 \mathrm{~K}$. Values of $367 \mathrm{kA} \mathrm{m}^{-1}, 1.78$ and $5.3 \times 10^{-6} \mathrm{~K}^{-1.78}$ have been used for $M_{\mathrm{s}}(0), \beta$ and $B$, respectively, to fit the simulated ZFC data above $175 \mathrm{~K}$ in an external field of $1.6 \mathrm{kA} \mathrm{m}^{-1}$

\begin{tabular}{|c|c|c|c|c|}
\hline \multirow[b]{2}{*}{ Sample } & \multicolumn{2}{|c|}{ Magnetic measurements } & \multicolumn{2}{|c|}{ MC simulations } \\
\hline & $\begin{array}{l}M_{\mathrm{S}}(0) \\
\left(\mathrm{kA} \mathrm{m}^{-1}\right)\end{array}$ & $\begin{array}{l}\text { Experimentxal } \\
\alpha\end{array}$ & $\begin{array}{l}\text { Number of } \\
\text { layers }\end{array}$ & Simulated $\alpha$ \\
\hline Dispersion & 355 & $-0.0053^{a}$ & - & $-0.0007(19)$ \\
\hline Monolayer & & & 1 & $0.0640(14)$ \\
\hline Thin array & 369 & 0.045 & 3 & $0.0538(18)$ \\
\hline Medium array & 367 & 0.036 & 6 & $0.0481(15)$ \\
\hline Thick array & 370 & 0.020 & 30 & $0.0343(20)$ \\
\hline & & & Bulk $(\infty)$ & $-0.0313(18)$ \\
\hline
\end{tabular}

${ }^{a}$ The negative value obtained for the dispersion, indicating a weak antiferromagnetic coupling between the dipole moments, is likely due to the presence of small aggregates of nanoparticles (see ESI $\dagger$ ). This is further supported by the low value for the remanent magnetization of the dispersion $\left(M_{\mathrm{R}} / M_{\mathrm{S}}=0.28\right)$ in Fig. 2, which is lower than the ideal value of 0.5 for randomly oriented monodomain particles with uniaxial anisotropy. ${ }^{41,42}$ 

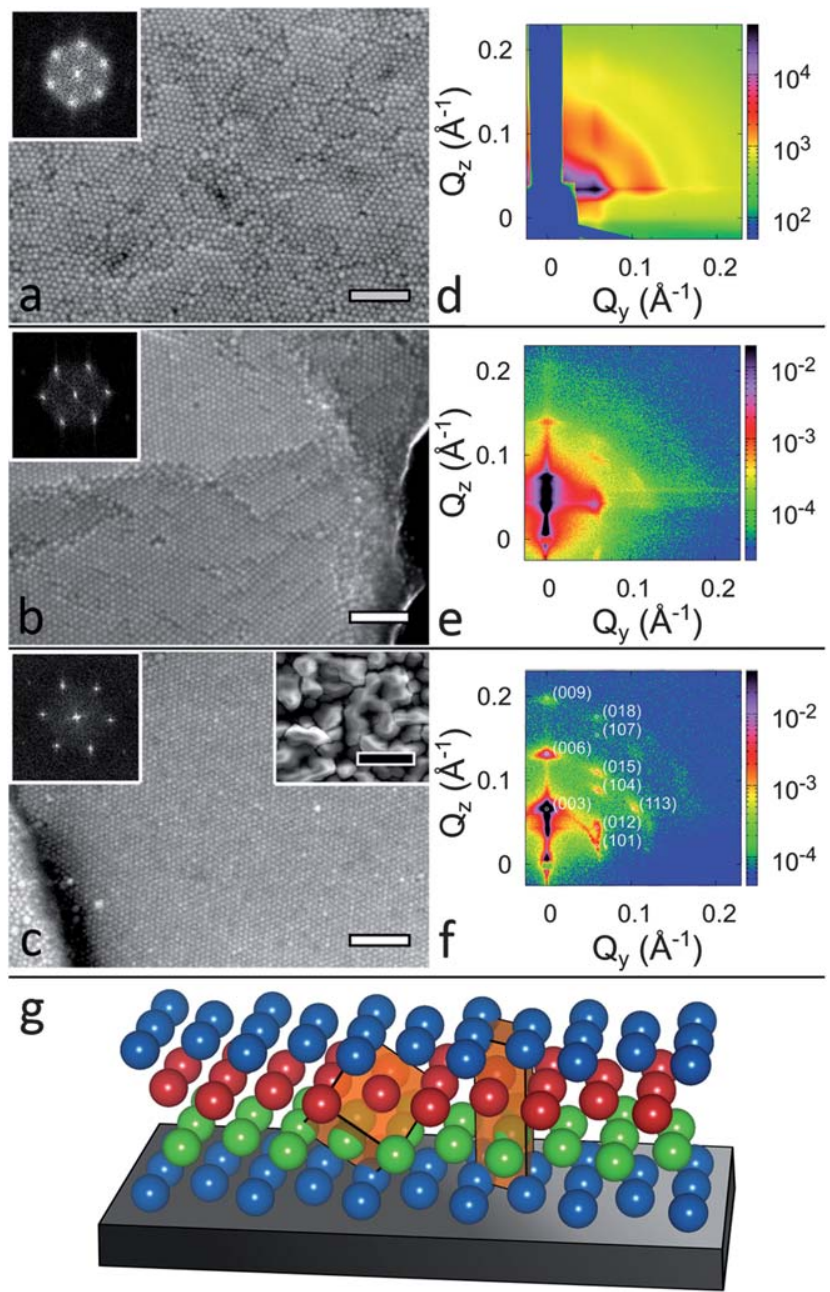

Fig. 1 SEM images and GISAXS patterns of the thin (a and $d)$, medium ( $b$ and $e$ ), and thick (with low-resolution inset) (c and f) arrays; respectively. (Scale bars: white $-100 \mathrm{~nm}$, black $-10 \mu \mathrm{m}$ ). (g) Structure model illustrating the orientation of the nanoparticle arrays on the substrate (black) with the primitive rhombohedral and fcc unit cells (indicated in orange, from right to left). The insets in the top-left corner $(a-c)$ are the FFT of the corresponding SEM image. X-ray pattern (d) was taken at the Soleil synchrotron source with a long beam stop, (e) and (f) at the Risø laboratory source with a circular beam stop.

(FM-like) coupling between the dipole moments in the arrays, increasing in strength with decreasing thickness and also enable a more in-depth study of the magnetic interactions in the framework of a mean-field model.

In a mean-field approach, the dipolar interaction field $H_{\mathrm{d}}$ due to neighboring nanocrystals is expressed as $H_{\mathrm{d}}=\alpha M$, where $M$ is the field-induced magnetization and $\alpha$ is the mean-field constant, a measure of the dipolar couplings in the arrays; it is negative for antiferromagnetic and positive for ferromagnetic couplings. A Bloch-like law was used to describe the temperature dependence of the monodomain nanoparticle magnetic moments; $m(T)=M_{\mathrm{S}}(T) V_{\mathrm{p}}=M_{\mathrm{S}}(0) V_{\mathrm{p}} \times\left(1-B T^{\beta}\right)$, where $M_{\mathrm{S}}$ is the nanocrystal saturation magnetization and $V_{\mathrm{p}}$ is the volume of a nanoparticle. $\beta=1.78 \pm 0.02$ and $B=5.3 \pm 0.6 \times$ $10^{-6} \mathrm{~K}^{-1.78}$ were determined from magnetization versus field measurements performed at different temperatures, together with high field magnetization versus temperature

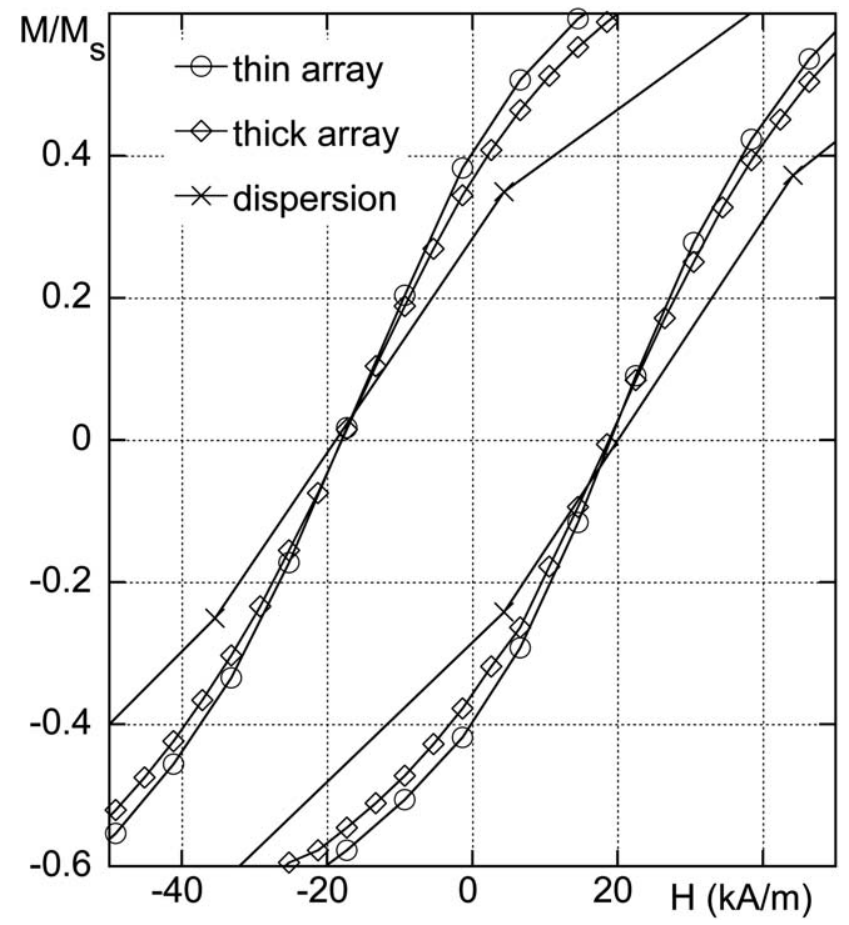

Fig. 2 Normalized magnetization $M / M_{\varsigma}$ versus the applied field $H$ (hysteresis loops) for the thin $(O)$ and thick $(\diamond)$ arrays and for the dispersion $(x)$ at $10 \mathrm{~K}$. The data have been corrected for an exchange bias effect, ${ }^{44}$ and the full hysteresis loops are available in the ESI. $†$ The lines are guides to the eye.

measurements (see ESI $)$ ). The fitted parameters are in good agreement with previously reported values for clusters of iron oxide nanoparticles. ${ }^{36,37}$ A Curie-Weiss law could then be used to fit the low-field inverse susceptibility data corresponding to the linear response regime:

$$
\frac{1}{\chi}=\frac{T-\alpha C(0)\left(1-B T^{\beta}\right)^{2}}{C(0)\left(1-B T^{\beta}\right)^{2}},
$$

where $C(0)=\frac{\mu_{0} V_{p}}{3 k_{\mathrm{B}}} M_{\mathrm{S}}{ }^{2}(0)$, using $\alpha$ and $M_{\mathrm{S}}(0)$ as fitting parameters. $\mu_{0}$ is the permeability of free space and $k_{\mathrm{B}}$ is the Boltzmann constant. The fits are shown in Fig. 3(b) and the extracted values for the fitting parameters are given in Table 2 . The $M_{\mathrm{S}}(0)$ values for the nanospheres are, as expected, lower than for bulk maghemite..$^{38}$ In general, differences in magnetic properties between nanoparticles and bulk materials are attributed to differences in the magnetic order between the surface and the core of the nanoparticles, and also to a lowered magnetization in the core itself. ${ }^{39,40}$

The value of $\alpha$ for the arrays increases with decreasing thickness, confirming that the decreasing finite thickness of the arrays enhances FM-like couplings. To the best of our knowledge, this is the first report of a thickness-dependent ferromagnetic coupling in ordered arrays of monodomain magnetic nanoparticles. However, it should be emphasized that the arrays do not exhibit long-range ferromagnetic order at low temperature. The reason for this becomes clear comparing the magnitudes of the dipolar interaction $\left(E_{\mathrm{d}}=-\mu_{0} \alpha M m\right)$ and the magnetic anisotropy $\left(E_{\mathrm{a}}=K V_{\mathrm{p}}\right.$, where $K$ is the anisotropy 

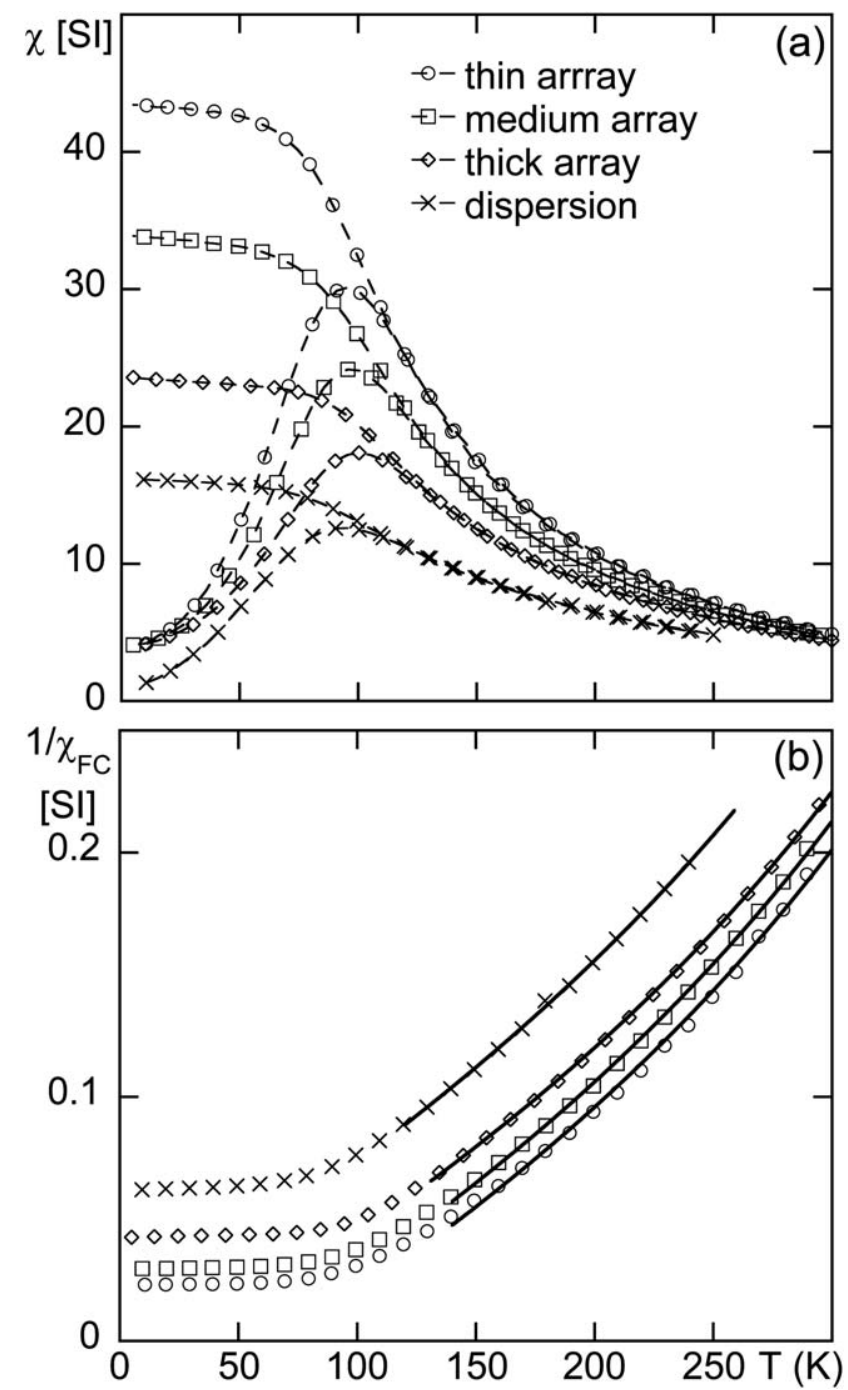

Fig. 3 Temperature-dependent in-plane susceptibility of the arrays and the dispersion ( $0.1 \mathrm{wt} \%$ ) of iron oxide nanocrystals under a low external field $(H=0.4$ $\mathrm{KA} \mathrm{m}^{-1}$ ). The susceptibility values are normalized with respect to the amount of iron oxide nanoparticles. (a) FC and ZFC susceptibility vs. temperature $T$ for the thin $(\bigcirc)$, medium $(\square)$, thick $(\diamond)$ arrays and the dilute dispersion $(\times)$. The dashed lines are guides to the eye. (b) Inverse FC susceptibility $1 / \chi_{F C} v$ s. temperature $T$ for the thin, medium, thick arrays and the dilute dispersion. The thick solid lines are fits to the high temperature part of the experimental data to a mean-field expression for the inverse susceptibility (eqn (1)).

constant) energies; $E_{\mathrm{a}}$ (see ESI $\dagger$ for details) is more than one order of magnitude larger than the dipolar interaction energy. This implies that at low temperature the particle magnetic moments will freeze along directions defined by the random distribution of easy magnetization directions before having the possibility to establish long-range magnetic order.

Each particle magnetic moment $\vec{m}_{\mathrm{i}}$ responds to a different local dipole field $\vec{H}_{\mathrm{d} \text {,i }}$, which is the sum of the dipolar interaction fields created by all the other magnetic moments in the array. The mean-field description assumes a uniform dipolar interaction field $\vec{H}_{\mathrm{d}}$ across the array, which is the average of all the local dipole fields. Thus, the decrease of the mean-field constant $\alpha$ with increasing thickness of the array indicates an increase in the directional variations of $\vec{H}_{\mathrm{d}, \mathrm{i}}$ ascribed to the formation of closed circuit-loops of dipole moments. In order to support this hypothesis, MC simulations were performed to estimate the amount of vortex states in the arrays.

The ZFC data for the dispersion and the arrays have been simulated by the MC method using the experimentally determined lattice parameters, temperature-dependent saturation magnetization and anisotropy constant (see ESI $\dagger$ for details).

Fig. 4 compares the experimental and simulated temperature-dependent ZFC susceptibility. The high-temperature part of the simulated data was fitted to eqn (1) to obtain the simulated mean-field coefficient $\alpha$. To obtain simulation data with good enough statistical precision, it was necessary to use an external field of intermediate magnitude $\left(H=1.6 \mathrm{kA} \mathrm{m}^{-1}\right)$.

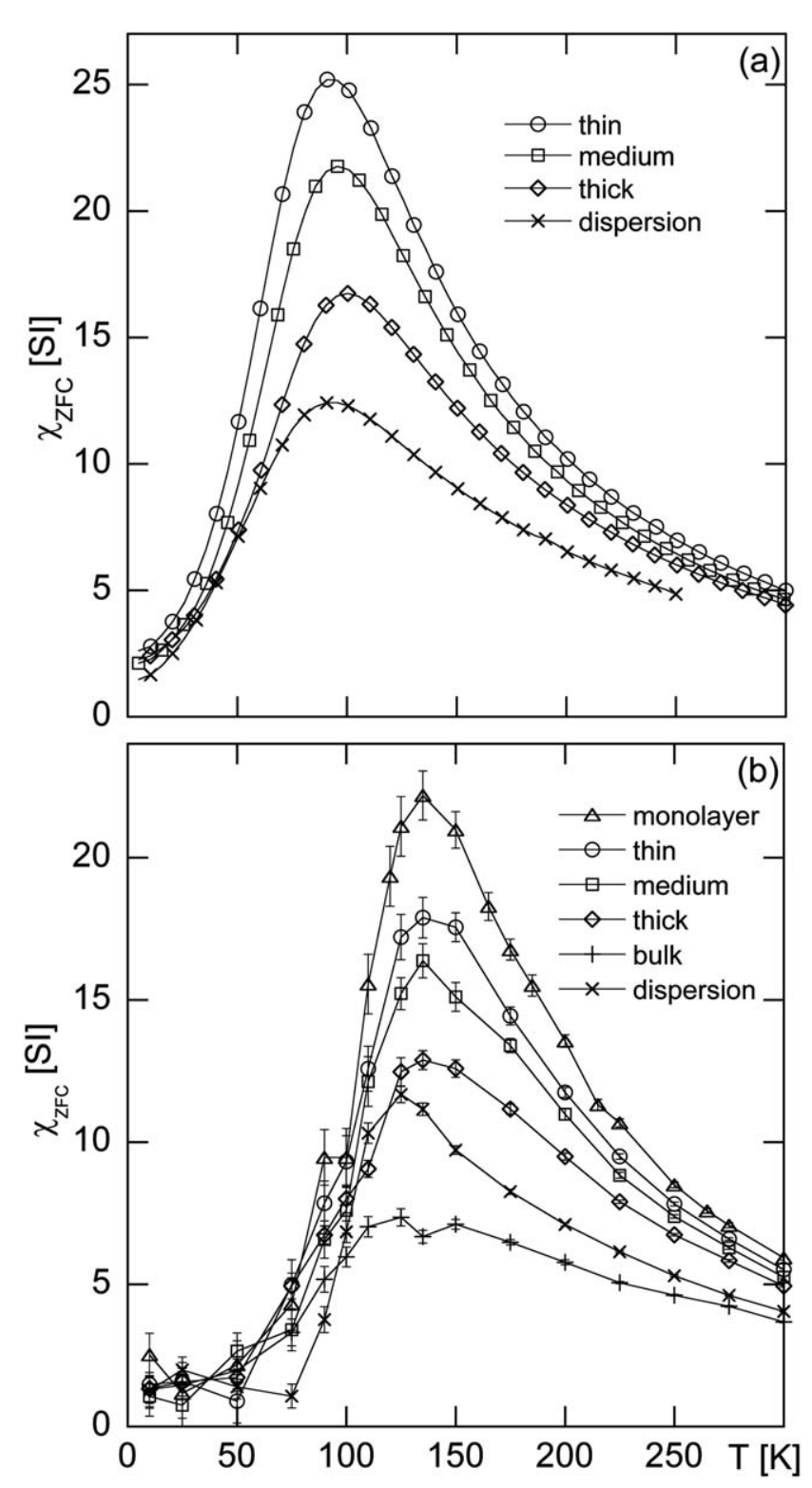

Fig. 4 ZFC in-plane susceptibility vs. temperature curves at intermediate field $\left(H=1.6 \mathrm{kA} \mathrm{m}^{-1}\right)$. The solid lines are guides to the eye. (a) Experimental curves for the arrays and the dispersion. (b) Curves obtained from the MC simulations. Each point is the average of 25 to 325 independent simulations, with a standard error shown by the error bars. 
A larger external field implies that deviations from a linear response will be observed at higher temperatures. Therefore, eqn (1) was fitted to the simulated data between $175 \mathrm{~K}$ and 300 K. As shown in Table 2, the simulated $\alpha$ values decrease with increasing thickness, in agreement with the experimental results. The simulated mean-field coefficients and the blocking temperatures are consistently higher than the experimental ones, which could be attributed to characteristics of the real arrays that are neglected in the simulations, e.g. the particle size distribution, the finite in-plane correlation length or the tilt of the arrays during the drying stage. In particular, the broader features observed in the experimental ZFC curves result from the distribution of particle sizes that the real arrays exhibit. Nevertheless the magnetic model comprising the Zeeman, anisotropy and dipolar energies qualitatively reproduces the thickness-dependent magnetic behavior of the arrays. In addition the simulated susceptibility and blocking temperature of the dispersion are lower than the values for the arrays, confirming that the dipolar interactions in the arrays induce a ferromagnetic coupling between the nanoparticle magnetic moments favoring a parallel alignment of the moments.

The simulated mean-field constant for the bulk arrays is negative, which corresponds to antiferromagnetic couplings. Energy calculations of infinite $f c c$ arrays of dipoles, excluding magnetic anisotropy, predict that the ground state is ferromagnetic at low temperature. ${ }^{21}$ However, it has been shown that finite boundary conditions, like those used for the simulations of the bulk arrays, can lead to an antiferromagnetic ordering of the dipoles. Moreover the crystallographic deviation of the trigonal lattice from an $f c c$ unit cell observed by GISAXS could induce antiferromagnetic couplings. ${ }^{43}$

In order to determine the mechanism responsible for the decrease in susceptibility with the array thickness, the remanent magnetization of the arrays was simulated by allowing inplane magnetically saturated arrays of dipoles to relax at $10 \mathrm{~K}$ at zero field. A good qualitative agreement is found between simulation and experimental results; the normalized remanent magnetization $M_{\mathrm{R}} / M_{\mathrm{S}}$ for both cases decreases with increasing thickness of the array and it is larger for the arrays in comparison to the dispersion, $c f$. Fig. 5(a).

The vorticity vector field shown in Fig. 5(b) for the equilibrium zero-field state at $10 \mathrm{~K}$ has been used to quantify the tendency of the dipoles to form closed-circuit loops. The relationship between the orientation of the magnetic closed-loops and the vorticity vector is illustrated in Fig. 6 . The average root sum square of the $\vec{V}_{\text {in }}$ and $\vec{V}_{\text {out }}$ components of the vorticity field has been averaged over all the dipoles in each simulation, and then averaged over several independent simulations. In order to determine the average orientation of the closed-flux loops, the ratio $2\left\langle V_{\text {in }}\right\rangle^{2} /\left\langle V_{\text {out }}\right\rangle^{2}$ was calculated. For an isotropic orientation of the magnetic closed-circuit loops, the ratio is 1 . The vorticity for the monolayer was not calculated due to the absence of a defined gradient of the magnetization in the $z$-direction.

The total vorticity of the arrays increases with the thickness, in good agreement with previous simulations. ${ }^{27}$ Thus, the vorticity and the remanent magnetization in Fig. 5 are inversely (a)

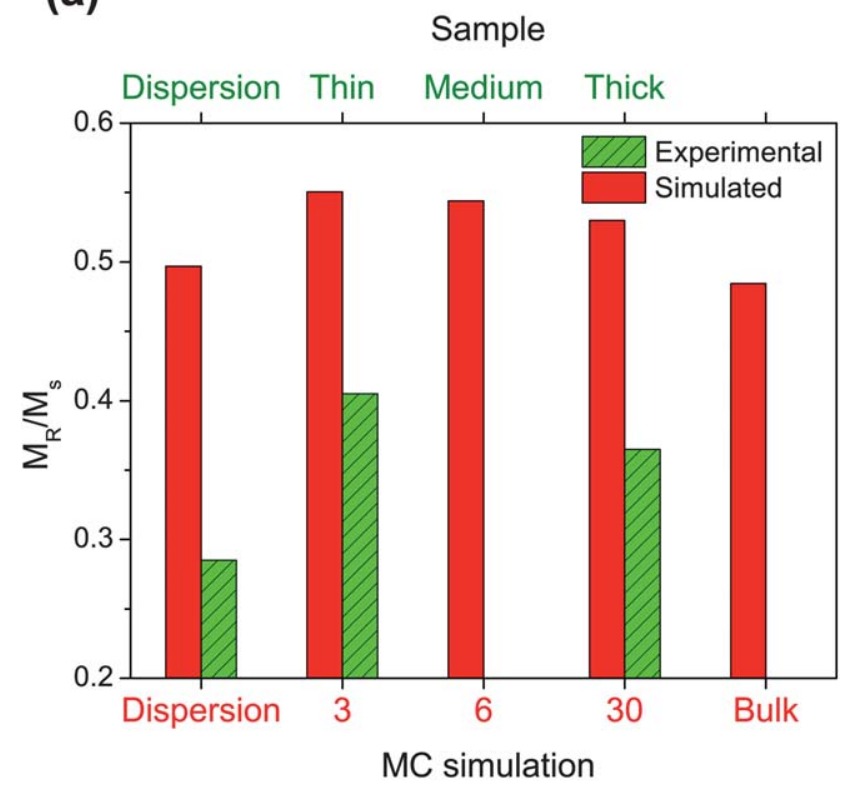

(b)

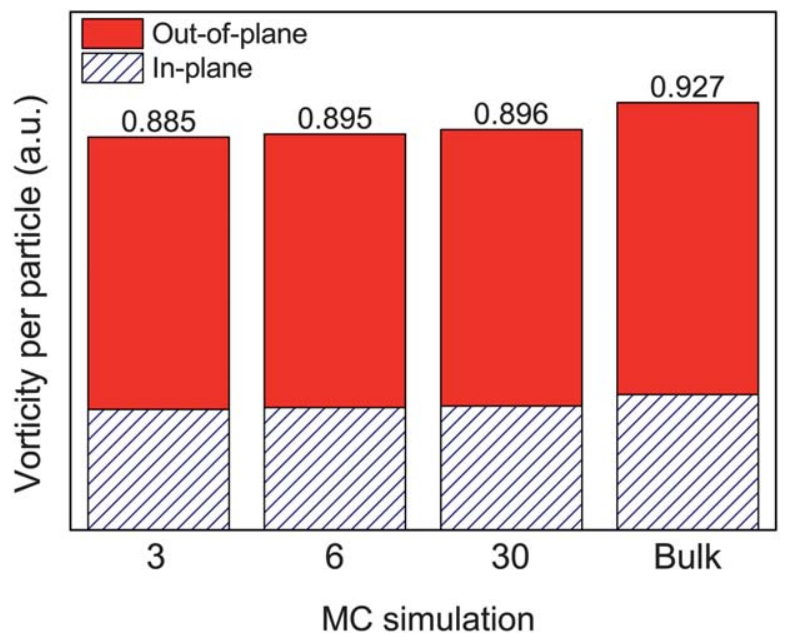

Fig. 5 Simulated remanent magnetization and vorticity of the arrays at $10 \mathrm{~K}$. (a) Remanent magnetization in the arrays $M_{R}$, normalized by the saturation magnetization $M_{S}$ at $10 \mathrm{~K}$. The corresponding experimental values for the dispersion as well as for the thin and thick arrays are included for comparison. (b) Average root sum square of the in-plane and out-of-plane components of the vorticity field, with respect to the surface of the substrate. The numbers represent the ratio of twice the squared in-plane contribution over the squared out-ofplane one.

correlated. Indeed the ferromagnetic correlations are associated with a low vorticity, so that the total vorticity increases with the thickness, whereas the mean-field constant $\alpha$ decreases (Table 2 ). The decrease of the ratio for the thin array (3 layers) suggests that the mechanism for the $2 \mathrm{D}$ to $3 \mathrm{D}$ crossover is a change in the orientation of the vortex states, which are oriented preferentially out-of-plane as the thickness decreases. The proportion of in-plane vorticity increases with the thickness, but an isotropic orientation of the vortex state is not observed in the bulk arrays, presumably due to the deviation of the array structure from a perfect $f c c$ lattice. 


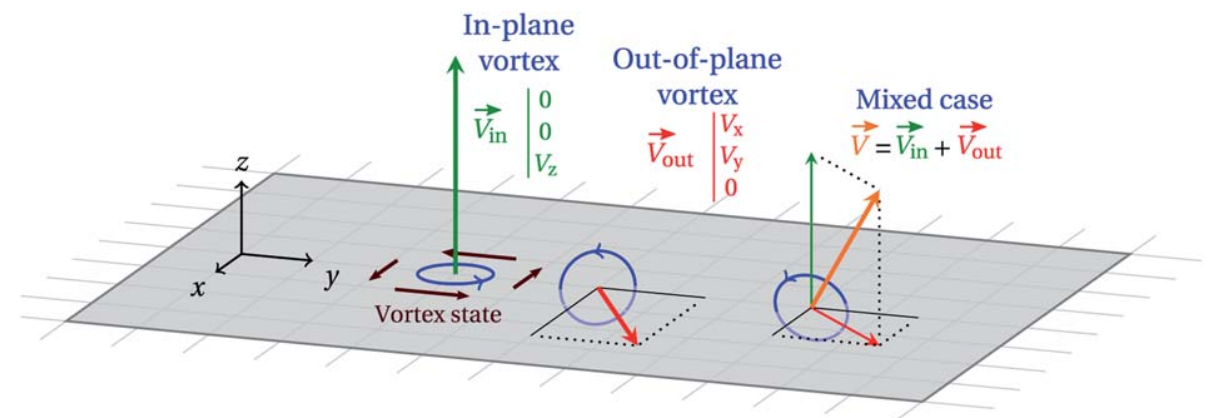

Fig. 6 Relationship between the orientation of the vortex states and the orientation of the vorticity vector

\section{Conclusions}

Collective magnetic properties of monodisperse nanospheres structured in arrays of different thicknesses have been characterized by temperature- and field-dependent magnetization measurements and compared with simulated curves obtained through MC simulations. The symmetry of the ordered arrays corresponds to a distorted $f c c$ lattice. The structure, experimentally determined saturation magnetization and anisotropy constant were used as input for the simulations, which could qualitatively reproduce the experimental results, with the right order of magnitude and trends in magnetic susceptibility. The combination of experimental and simulation results showed that magnetic dipolar interactions induce an effective ferromagnetic coupling in thin arrays. The coupling decreases in strength with increasing thickness of the array, to finally switch to antiferromagnetic coupling in the bulk limit. We find that the decrease of the ferromagnetic coupling relates to an increase of the vorticity in the simulated arrays. The mechanism of the $2 \mathrm{D}$ to 3D crossover was identified as a thickness-dependent orientation of the magnetic vortex states. The orientation of the vortex states becomes more isotropic as the thickness of the arrays increases. Magnetic anisotropy prevents long-range magnetic order from being established at low temperature and the nanoparticle magnetic moments instead freeze along directions defined by the distribution of easy magnetization directions.

\section{Acknowledgements}

The authors would like to thank the Swedish Research Council (VR) and the Knut and Alice Wallenberg Foundation (KAW) for financial support, the SOLEIL synchrotron for provision of beam time at SWING, the National Supercomputer Centre (Linköping, Sweden) for providing computation resources, Doc. Jekabs Grins (Stockholm University) for performing the Rietveld refinement and Dr Ulrich Rücker for helpful discussions.

\section{References}

1 S. Sun, C. B. Murray, D. Weller, L. Folks and A. Moser, Science, 2000, 287, 1989-1992.

2 J. Martín, J. Nogués, K. Liu, J. Vicent and I. K. Schuller, J. Magn. Magn. Mater., 2003, 256, 449-501.
3 M. P. Pileni, J. Phys. Chem. B, 2001, 105, 3358-3371.

4 S. Liu and Z. Tang, J. Mater. Chem., 2010, 20, 24-35.

5 D. V. Talapin, J.-S. Lee, M. V. Kovalenko and E. V. Shevchenko, Chem. Rev., 2010, 110, 389-458.

6 A. P. Alivisatos, Science, 1996, 271, 933-937.

7 C. B. Murray, C. R. Kagan and M. Bawendi, Annu. Rev. Mater. Sci., 2000, 30, 545-610.

8 C. R. Kagan, C. B. Murray and M. G. Bawendi, Phys. Rev. B, 1996, 54, 8633-8643.

9 C. Collier, R. Saykally, J. Shiang, S. Henrichs and J. Heath, Science, 1997, 277, 1978-1981.

10 C. T. Black, C. B. Murray, R. L. Sandstrom and S. Sun, Science, 2000, 290, 1131-1134.

11 S. I. Woods, J. R. Kirtley, S. Sun and R. H. Koch, Phys. Rev. Lett., 2001, 87, 137205.

12 P. Poddar, T. Telem-Shafir, T. Fried and G. Markovich, Phys. Rev. B, 2002, 66, 060403.

13 S. Yamamuro, D. F. Farrell and S. A. Majetich, Phys. Rev. B, 2002, 65, 224431.

14 M. Georgescu, M. Klokkenburg, B. H. Erné, P. Liljeroth, D. Vanmaekelbergh and P. A. Zeijlmans van Emmichoven, Phys. Rev. B, 2006, 73, 184415.

15 A. Ahniyaz, Y. Sakamoto and L. Bergström, Proc. Natl. Acad. Sci. U. S. A., 2007, 104, 17570-17574.

16 M. Georgescu, J. L. Viota, M. Klokkenburg, B. H. Erné, D. Vanmaekelbergh and P. A. Zeijlmans van Emmichoven, Phys. Rev. B, 2008, 77, 024423.

17 K. Yamamoto, S. A. Majetich, M. R. McCartney, M. Sachan, S. Yamamuro and T. Hirayama, Appl. Phys. Lett., 2008, 93, 082502 .

18 O. Kasyutich, R. D. Desautels, B. W. Southern and J. van Lierop, Phys. Rev. Lett., 2010, 104, 127205.

19 H. Singh, P. E. Laibinis and T. A. Hatton, Nano Lett., 2005, 5, 2149-2154.

20 K. Nakata, Y. Hu, O. Uzun, O. Bakr and F. Stellacci, Adv. Mater., 2008, 20, 4294-4299.

21 J. M. Luttinger and L. Tisza, Phys. Rev., 1946, 70, 954-964.

22 V. J. Russier, Appl. Phys., 2001, 89, 1287-1294.

23 P. Politi, M. G. Pini and R. L. Stamps, Phys. Rev. B, 2006, 73, 020405.

24 F. Luis, F. Petroff, J. M. Torres, L. M. García, J. Bartolomé, J. Carrey and A. Vaurès, Phys. Rev. Lett., 2002, 88, 217205. 
25 J. Pereira Nunes, M. Bahiana and C. Bastos, Phys. Rev. E, 2004, 69, 056703.

26 K. N. Trohidou and M. Vasilakaki, in Applications of Monte Carlo Method in Science and Engineering, ed. S. Mordechai, InTech, 2011, ch. 20.

27 P. R. Arias, D. Altbir and M. Bahiana, J. Phys.: Condens. Matter, 2005, 17, 1625-1633.

28 G. Salazar-Alvarez, J. Qin, V. Šepelák, I. Bergman, I. Vasilakakis, K. Trohidou, J. Ardisson, W. Macedo, M. Mikhaylova, M. Muhammed, M. Baró and J. Nogués, J. Am. Chem. Soc., 2008, 130, 13234-13239.

29 S. Disch, E. Wetterskog, R. Hermann, G. Salazar-Alvarez, P. Busch, L. Bergström, T. Brückel and S. Kamali, Nano Lett., 2011, 11, 1651-1656.

30 L. Häggström, S. Kamali, T. Ericsson, P. Nordblad, A. Ahniyaz and L. Bergström, Hyperfine Interact., 2008, 183, 49-53.

31 J. Kehres, J. W. Andreasen, F. C. Krebs, A. M. Molenbroek, I. Chorkendorff and T. Vegge, J. Appl. Crystallogr., 2010, 43, 1400-1408.

32 D. Kechrakos and K. Trohidou, Appl. Phys. Lett., 2002, 81, 4574-4576.

33 C. Papadakis, P. Busch, D. Posselt and D.-M. Smilgies, in Advances in Solid State Physics, ed. B. Kramer, Springer, Berlin, Heidelberg, 2004; vol. 44, pp. 327-338.

34 T. Hanrath, J. J. Choi and D.-M. Smilgies, ACS Nano, 2009, 3, 2975-2988.
35 X. M. Lin, H. M. Jaeger, C. M. Sorensen and K. J. Klabunde, J. Phys. Chem. B, 2001, 105, 3353-3357.

36 P. V. Hendriksen, S. Linderoth and P.-A. Lindgård, Phys. Rev. $B, 1993,48,7259-7273$.

37 C. Johansson, M. Hanson, M. S. Pedersen and S. Mørup, J. Magn. Magn. Mater., 1997, 173, 5-14.

38 X. Batlle, N. Pérez, P. Guardia, O. Iglesias, A. Labarta, F. Bartolomé, L. M. García, J. Bartolomé, A. G. Roca, M. P. Morales and C. J. Serna, J. Appl. Phys., 2011, 109, $07 \mathrm{~B} 524$.

39 S. Disch, E. Wetterskog, R. P. Hermann, A. Wiedenmann, U. Vainio, G. Salazar-Alvarez, L. Bergström and T. Brückel, New J. Phys., 2012, 14, 013025.

40 K. L. Krycka, R. A. Booth, C. R. Hogg, Y. Ijiri, J. A. Borchers, W. C. Chen, S. M. Watson, M. Laver, T. R. Gentile, L. R. Dedon, S. Harris, J. J. Rhyne and S. A. Majetich, Phys. Rev. Lett., 2010, 104, 207203.

41 E. C. Stoner and E. P. Wohlfarth, Philos. Trans. R. Soc. London, 1948, 240, 599-642.

42 B. D. Cullity and C. D. Graham, Introduction to Magnetic Materials, Wiley-IEEE Press, 2nd edn, 2008, p. 568.

43 P. Panissod and M. Drillon, in Magnetism: Molecules to Materials IV, ed. J. S. Miller and M. Drillon, Wiley-VCH, 1st edn, 2002, ch. 7, pp. 233-270.

44 J. Nogués, J. Sort, V. Langlais, V. Skumryev, S. Suriñach, J. Muñoz and M. Baró, Phys. Rep., 2005, 422, 65-117. 